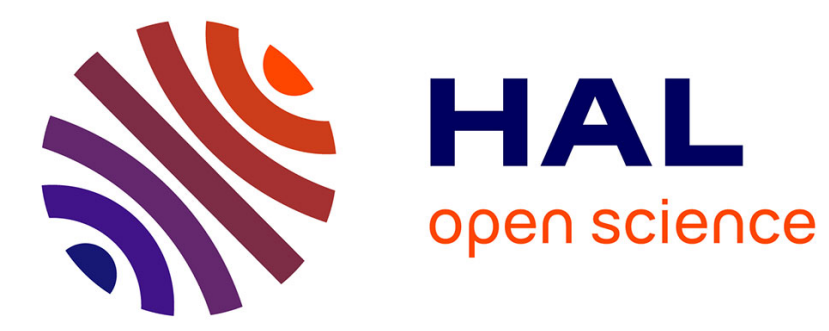

\title{
Transvenous path finding in cardiac resynchronization therapy
}

Jean-Louis Coatrieux, Alfredo I. Hernandez, Philippe Mabo, Mireille Garreau, Pascal Haigron

\section{- To cite this version:}

Jean-Louis Coatrieux, Alfredo I. Hernandez, Philippe Mabo, Mireille Garreau, Pascal Haigron. Transvenous path finding in cardiac resynchronization therapy. 3rd International Workshop on Functional Imaging and Modeling of the Heart, Jun 2005, Barcelona, Spain. pp.236-245. inserm-00530419

\section{HAL Id: inserm-00530419 https://www.hal.inserm.fr/inserm-00530419}

Submitted on 28 Oct 2010

HAL is a multi-disciplinary open access archive for the deposit and dissemination of scientific research documents, whether they are published or not. The documents may come from teaching and research institutions in France or abroad, or from public or private research centers.
L'archive ouverte pluridisciplinaire HAL, est destinée au dépôt et à la diffusion de documents scientifiques de niveau recherche, publiés ou non, émanant des établissements d'enseignement et de recherche français ou étrangers, des laboratoires publics ou privés. 


\title{
Transvenous Path Finding In Cardiac Resynchronization Therapy
}

\author{
Jean Louis Coatrieux ${ }^{1}$, Alfredo I. Hernández ${ }^{1}$, Philippe Mabo ${ }^{2}$, Mireille Garreau ${ }^{1}$, \\ and Pascal Haigron ${ }^{1}$ \\ ${ }^{1}$ Laboratoire Traitement du Signal et de l'Image, INSERM, Université de Rennes 1, \\ Campus de Beaulieu, 35042 Rennes Cedex, France \\ http://www.ltsi.univ-rennesl.fr \\ ${ }^{2}$ Centre Cardio-Pneumologique, CHU Pontchaillou, 35033 Rennes, France
}

\begin{abstract}
Cardiovascular diseases are a major health concern all over the world and, especially, heart failure has gained more importance in the recent years. Improving diagnosis and therapy is therefore critical and among the several resources at our disposal, implantable devices is expected to have a better rate of success. This paper is focused on two topics: (i) our views of the main challenges to face in order to reach these objectives and (ii) a specific target regarding the pose of leads for multisite pacemakers by means of virtual endoscopy pre-operative planning and path finding throughout the coronary venous tree.
\end{abstract}

\section{Introduction}

The cardiovascular disorders remain the most important cause of death in all countries. They cover a wide spectrum of causes among which coronary artery and cardiac valve diseases, abnormal excitation-contraction coupling or heart failure. Electrophysiological pathologies are among the most deeply investigated for a long time. They include cardiac arrhythmia and myocardial ischemia which both may originate from very distinct locations and have many underlying expressions (ectopic foci, spiral-like wavefronts, conduction blocks, ..). In all cases, early diagnosis must be based on a full exploration of the whole heart instead of focusing on the left ventricle. However, any improvement at the diagnosis stage will be of limited interest if subsequent sound therapies are not available. Advances have been made over years in the design of drugs but some of them have been associated with side effects and negative outcomes. Technological solutions rely on Cardiac Resynchronization Therapy and Radiofrequency Ablation. They both share, to be efficient, a lot of concerns regarding the definition of target sites where abnormal patterns are observed, the consequences of which are acute and overall dysfunctions in the heart pumping. They require advanced imaging techniques for their localization and for assisting the physician before, during and after the interventions.

This paper is focused on Cardiac Resynchronization Therapy (CRT) aimed at restoring the contractile coordination in hearts with severe heart failure (HF), sinus rhythm and ventricular conduction delay. It is performed by stimulating both the right and the 
left ventricles, pacing them simultaneously or with a small delay. Several clinical trials have shown that this technique is beneficial for acute as well as chronic disorders, improving the heart's performance, the capacity of the patient for exercise and reducing the mortality from heart failure, [Cazeau, 2001], [Leclercq, 2002], [Kass, 2003]. However, these studies point out that the main issue remains to identify and assess the most effective pacing sites in order to reduce the percentage of nonresponding patients which may reach up to 25 to $30 \%$ of recipients. They have also shown that LV-lead positioning (either LV-only pacing or biventricular pacing) is without contest the most challenging task to carry out.

Our objective is therefore to better prepare the placement of CRT leads using the new Multislice Computed Tomography (MSCT) capabilities in imaging the heart. Section 2 provides a brief overview of the multiple challenges we must address at long range to understand how the major components (electrical, mechanical, etc.) work together and are regulated under normal and abnormal physiological conditions. Section 3 brings more clues on the CRT issues in pre-operative context. Section 4 reports some preliminary results achieved by using virtual endoscopy and a few perspectives are discussed in Section 5.

\section{The overall heart picture}

The key dimensions [Coatrieux, 2004] for further advances in clinical diagnosis and therapy are reported figure 1 . Only a few of them, that we consider as major issues to deal with, will be detailed here.

The identification of the disorder through non-invasive data recording is the first stage to go through. The most generic and relevant tool remains standardized ECG (or esophageal ECG) which can be coupled to clinical observations (symptoms, past history), impedance measurements (cardiac output), phonocardiogram (PCG)... Body Surface Potential Mapping (with or without the so-called direct-inverse problem solving) has been shown superior to the conventional 12-lead ECG for non-invasively identifying the sites of earliest endocardial activation and the further spread through the ventricles. However, all these resources only give first assumptions on the abnormal behaviours that are observed and their potential localizations.

The integration of multimodal imaging data is another critical issue [Roux, 1997]. It starts with the diagnosis tools providing the 2D, 3D and 4D elements to capture local, regional and global characteristics required to determine the morphological and functional patterns of the heart, either normal or abnormal. The progress in ultrasound techniques, and in Multislice CT allows now to acquire 3D time image sequences with high contrast and spatio-temporal resolutions. The major problems, beyond spatio-temporal registration methods aimed at deriving a common coordinate system, are to extract quantitative features that can be physically and physiologically interpreted with a proper anatomical reference. Accurate and reliable segmentation methods, fulfilling the time computation constraints in clinical practice, with robust motion estimation algorithms and perfusion parameters have to be combined in a sound information processing frame in order to get a full view of the status of the heart. The constraints imposed in intra-operative environment, the therapeutic nature of the in- 
tervention, are even more demanding due to the real-time responses required for registration, detection, guidance of instruments, etc.

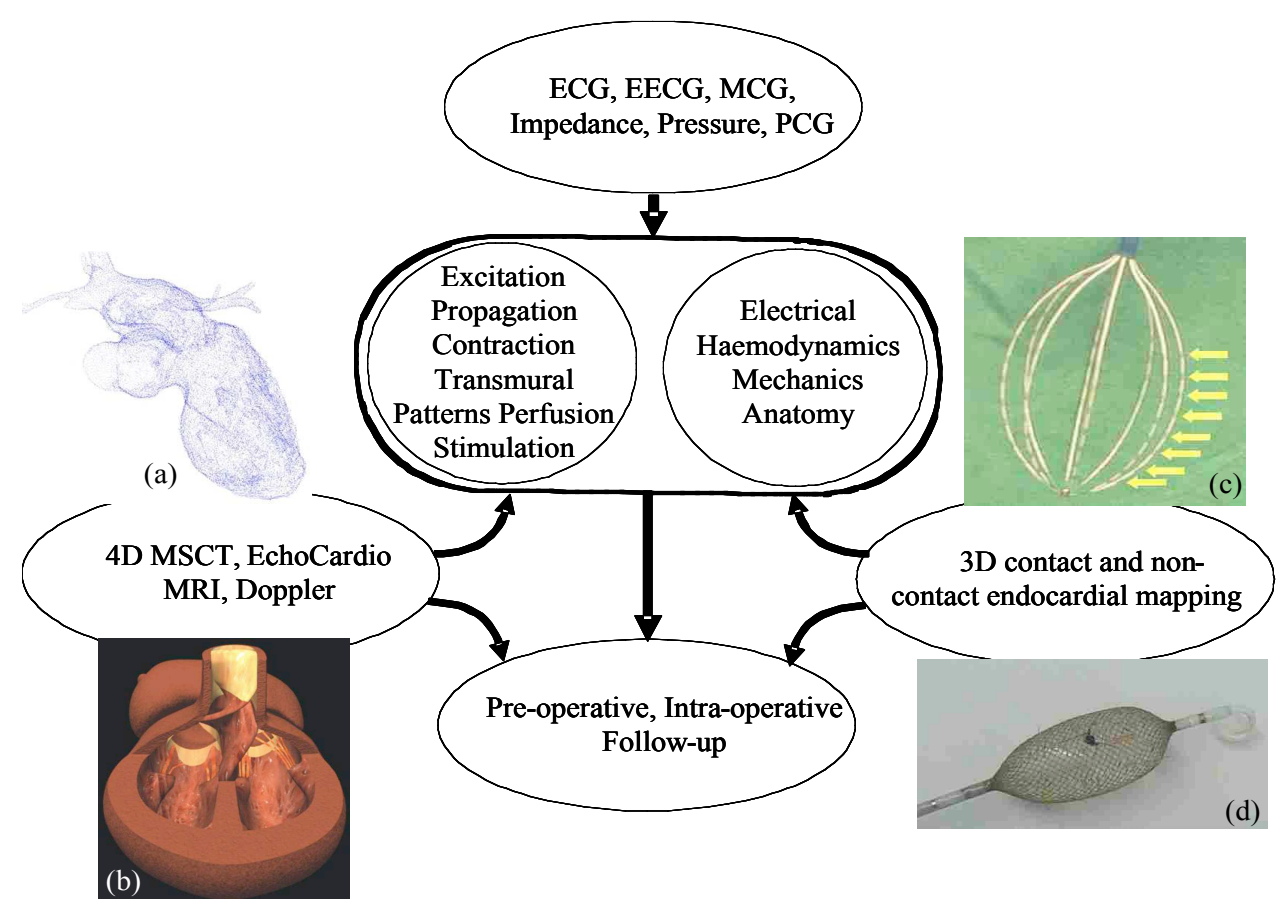

Fig. 1. Clinical diagnosis and therapy. Picture (a) is from [Garreau, 2004]. Picture (b) is from [Schleich, 2002]. Pictures (c) and (d) are from [de Boer, 2000].

Electrical mapping [Gepstein, 1997] [Schilling, 1998] [De Bakker, 2000] is a relevant complement of the imaging sources for electrophysiological tracking. The availability of catheter-based 3D non fluoroscopic contact (Carto, Biosense, or Constellation, Boston Scientific, Inc) and noncontact mapping (Ensite 3000, Endocardial Solutions, Inc) techniques allow in vivo assessment of the activation sequence with a relatively high spatial resolution. If the benefits of these techniques are clearly established in terms of electrophysiological insights, they have the inconvenient to be invasive, expensive and to increase the time duration of the exploration, and as such put more clinical demands.

The physiopathological in-silico modelling of the heart capable to fuse together the patient specific features (i.e electrical, mechanical and perhaps more importantly the electromechanical, mechanochemical, etc.) with the corresponding anatomical structures into generic models integrating the last data obtained through in-vitro, ex-vivo and in-vivo experiments is perhaps the grand challenge for tomorrow. A lot of efforts have been devoted to the restitution of the electro-physiological activity of the heart and two main model families can be distinguished (refer to [Bardou, 1996] [Virag, 2001] for full references): i) Simplified models, which are limited to the simulation of an action potential waveform, without taking into account any sub-cellular process, 
such as the Fitzugh-Nagumo's model (which was later improved by Aliev and Panfilov) or the model proposed by van Capelle and Durrer and ii) Electrophysiologically detailed models: which are based on the Hodgkin-Huxley approach for modeling ionic currents. A variety of models have been proposed for the later type, with increasing levels of detail and for specific myocardial tissues (i.e. ventricular, atrial, or Purkinje myocites). Two significant examples, for the ventricular myocite, are: the Beeler and Reuter's model, developed in 1977 which introduced the dynamics of internal calcium concentration and the models proposed by Luo and Rudy, with an increased number of ionic currents and a more detailed calcium dynamics. Bidimensional networks of cells can be built, where each node is described by one of these models and an extension of the cable equation is used to couple them. This approach has been readily applied since the 80 s to model myocardial propagation.

More recently, large-scale electrical models have been developed [Noble, 1997] [Quan, 1998]. Some of these models have been mapped to 3D anatomical data [Hunter, 1996], [Rudy, 1995] [Sermesant, 2004] but the key issue remains the inverse problem, i.e. the identification of the system from the current observed data. However, even if it is not out of reach, we are still far to deal with the full complexity of cardiac mechanisms. To just take an example, the excitation-contraction coupling, which refers to the physiological processes linking myocite depolarisation and contraction, involves many structural and regulatory proteins whose nature and function are just emerging [Bers, 2002].

Merging the multifunctional models we need to face electrical, mechanical, hemodynamic facets, at different scales, distinct supports, time dynamics with the multimodal data that we have at our disposal, would directly impact our capability to diagnose and care.

Further advances should rely on the design of intelligent devices, implantable or not, able to handle the several variables required, with both real-time recording, processing, stimulation capabilities. Along this path, recent technological breakthroughs of implantable devices have been achieved: they concern biventricular pacing (or CRT), cardioverter-defibrillator (ICD, Implantable Cardioverter Defibrillator) or joint device (CRT-ICD).

The last fundamental component we wish to highlight concerns the pre-operative planning, intra-operative assistance and post-operative follow-up. With the advances in medical imaging, image processing and rendering, almost over three decades, the concept of computer-assisted surgery has emerged with the aim to reduce the duration of interventional procedures, make them more successful and secure [Taylor, 1996]. Many achievements have been reported from the early biopsy applications in the $80 \mathrm{~s}$ based on simple instruments, straight line trajectories into rigid tissues. Image-guided therapies, with several marketed products, now go well beyond surgical procedures and address soft tissue applications as well as moving organs like the heart. They are of relevance for mitral valve replacement, ablation and pacing procedure. They share a number of methodological, technical and clinical features but also include several specific components, the main one for pacing being related to the venous coronary tree exploration that will be examined in the next paragraph. 


\section{Cardiac Resynchronization Therapy}

In 1998, a way to overcome the problem of multisite pacing by using a transvenous technique has been proposed [Daubert, 1998], permitting to stimulate the left ventricle on a long-term basis. The overall procedure consists in positioning endocardial leads in the right atrium and the right ventricle, the left ventricle being paced via a lead passed through the coronary sinus to an epicardial vein on the free wall of the left ventricle. The implant success rate, in between 85 and 92\%, although already high, is limited by the impossibility to access the target vein for lead placement, incorrect or suboptimal pacing site selection and possible electrode displacements [Alonzo, 2001], [Abraham, 2002].

The main objective is thus to assist cardiologists in improving and securing the implant techniques. From a clinical standpoint, it will rely on the study of the patient's coronary anatomy to define the target veins, to confirm their accessibility and to minimize the implant time. The pre-operative assistance, defined as Virtual Navigation [Haigron, 2004], will consist here to:

- Navigate through the patient's coronary venous tree so as to define the potential access paths for the pacing leads.

- Define which catheter and guide type should be used (with different diameters and curvatures, for example).

- Better define the optimal pacing site, based on the anatomo-functional information and on the electro-mechanical models of the cardiac activity.

Up to now, to our knowledge, there was no image-based planning of the implant procedure because no imaging source was capable to provide a full, 3D, time image sequence access to the venous tree of the heart. The pose of CRT is still directly performed, after the decision to implant a CRT, by using 2D venous coronary X-ray which leads to a partial and limited access to 3D anatomy.

The availability of MSCT is dramatically changing this situation: this 4D functional imaging CT Scanners can be used to obtain the basic structural and functional features required to achieve an optimal CRT planning. The LV can be paced transvenously through a subclavian vein, going successively via the cava vein, the right atrium, the coronary sinus and the great vein. The target location is a lateral or posterolateral vein. If lateral vein catheterization failed or in the case of poor pacing threshold, the $\mathrm{LV}$ lead is inserted into the great cardiac vein to pace the anterobasal wall or into the mid cardiac vein to pace the inferoapical region. Specifically designed coronary sinus leads are used. The injection of contrast medium in conventional X-ray angiography allows viewing the venous tree to be explored but it remains difficult to visually analyse due to the backward blood flow.

\section{Looking pre-operatively for a Left Ventricular path: preliminary results}

The procedure that has been worked out consists to select spatial sequences among 8 angio-scanners at our disposal (with minimal motion artefacts). The data were acquired on a Siemens Somatom volume zoom 4 detectors. Identical protocols were used with the following acquisition parameters: collimation of $0.6 \mathrm{~mm}$, table dis- 
placement of $1.5 \mathrm{~mm} /$ rotation, reconstruction increment of $0.6 \mathrm{~mm}$, size of the matrix $512 \times 512$ with about 250 slices and a pixel size from $0.33 \times 0.33$ to $0.4 \times 0.4 \mathrm{~mm}$. The resolution is 12 bits and the slice thickness of $1.25 \mathrm{~mm}$.

In this section, we present one example of navigation which has been prepared following the anatomical pathway required for the implantation of the left lead of a biventricular pacemaker. As emphasized from the beginning, this path is the one that implies the highest difficulty along the pacemaker implantation procedure. The principles of the virtual navigation have been reported elsewhere [Haigron, 1996]. The interactive procedure has been retained here. It consists for the physician to position the virtual sensor inside the object of interest, to define the viewing direction and to set the detection threshold. These tasks are performed by using three orthogonal planes commonly used in marketed workstations. In short, the algorithm used for the image computation relies on a rough detection of the inner surface of the vessels, a linear interpolation of the subvolume around this point, a refined detection with subvoxel accuracy, the computation of the surface normal for shading. Experiments have been conducted to evaluate the influence of the threshold in different data volumes, for different objects and locations. It appears that its setting is not difficult to control by the user and provides good results within the window 70-120 Hounsfield units. In addition, the method performs a semi-automatic analysis of the computed image in such a way that the vascular branches can be identified, their locations stored and paths constructed with a possible backward exploration. Quantitative features, going from local lumen diameters, centerline positions, calcification volume if any, angles at bifurcations, are available during the navigation.

The virtual sequences that have been defined correspond to hundreds of computed images that would need to be displayed as video records. Only a few of them are depicted Figure 2 in order to show the high quality that can be reached. It must be emphasized that the size of the distal veins is very small (a diameter approximately of 34 voxels or even less) and that, even in such situations, the current tools at our disposal behave well. The resulting 3D trajectories of several paths, using a MIP display, are described Figure 3 for different viewpoints.

The feasibility of the search for candidate paths is the first point that is assessed. The image quality is high enough to show details as well as major information about the environment into which the navigation takes place.

However, the search for some structures is not always easy. For example, when the virtual sensor is located in the atrium, finding the coronary sinus can take some time. This is also the case when we are looking for a specific vein. From our experiments, several reasons can be invoked to explain that: (i) we must be fully familiar with the anatomy of the venous network; (ii) a learning curve is necessary before decoding the MSCT data which are providing a lot of insights in the heart but, conversely, lead to complex reading; (iii) the virtual images have also to be practiced and local views must be referred to global views simultaneously in order to facilitate the exploration.

The preliminary experiments conducted with our clinical partners point out that this pre-operative planning represents a relevant step for CRT. The time required to define the candidate paths may reach up to 10 minutes but with a learning phase it is foreseen to be significantly reduced. A quantitative validation is in progress to estimate the reduction gained in intra-oprative time and consequently in terms of irradiation benefits. Finding the candidate pathways, verifying if the stimulation site is accessi- 
ble, estimating feasibility of the implantation, learning the gestures to be carried out, selecting the proper instruments, etc. are all major components. Thus, they allow reducing risk for the patient, optimising and facilitating the implantation procedure and subsequently, reducing the time of medical personal and operating rooms required.
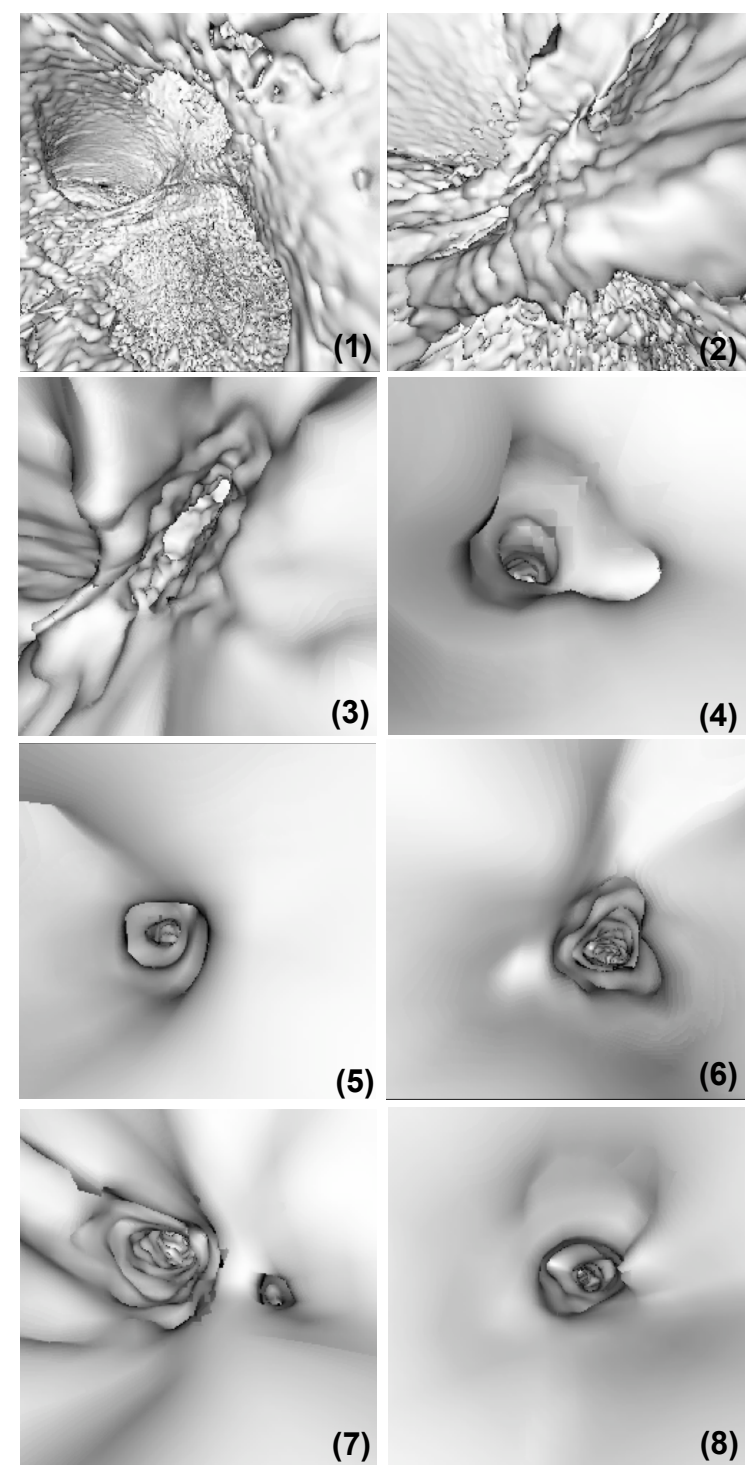

Fig. 2. Some virtual images computed during the navigation and showing the right atrium (1, 2 ), the postero-lateral and Marshall's veins $(4,5)$, the coronary sinus $(3,6)$, the great vein and the lateral veins $(7,8)$. The positions $(1-8)$ are also reported in figure 3 . 

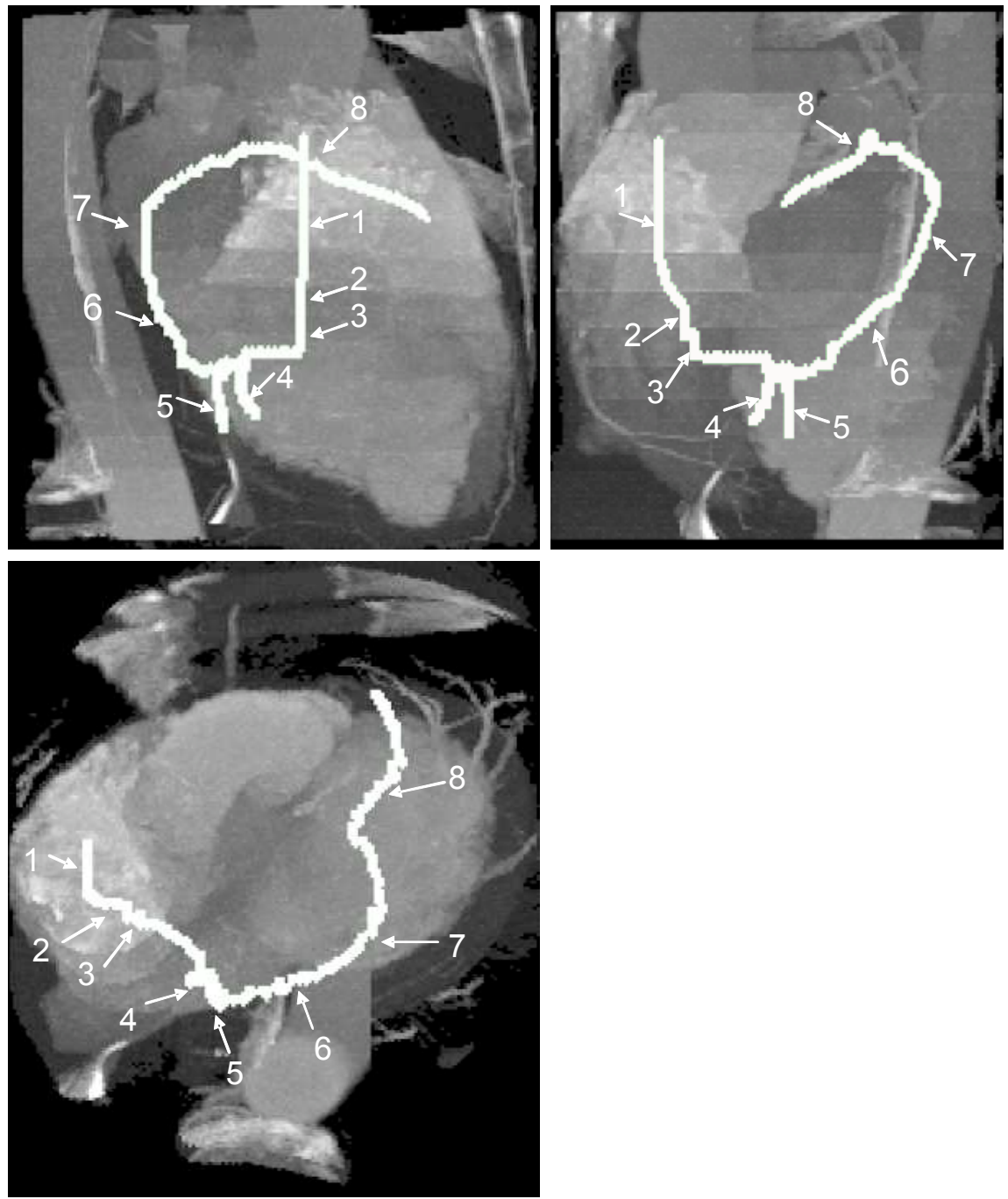

Fig. 3. Resulting 3D trajectories (in white) visualized using MIP from different points of view (lateral, apical and posterior). The ribs and other anatomical structures surrounding the heart have been suppressed using an appropriate ROI.

From the engineering standpoint point, the genericity of the solutions, early proposed for navigating into peripheral vessel networks and coronary arteries (where stenoses and calcifications may be present), has been demonstrated. The thresholding criteria involved in detection, interactively defined for a given data set, have been systematically studied and are not sensitive (i.e the interval of values is sufficiently large to provide enough robustness). 


\section{Conclusion}

The conjunction of electrical data, morphological and mechanical behaviour is very likely a source of additional progress. Insights into electromechanical coupling should improve the understanding of local, regional and global abnormalities and the localization of optimal stimulation or ablation sites. The present work was devoted to a subproblem in this overall frame: the pre-operative assistance of biventricular pacing based on a transvenous technique using the new possibilities offered by Multi-Slice Computed Tomography. These images are difficult to explore due to the many structures that are enhanced and to the complexity of the venous tree, close and strongly intermingled with the arterial network, and composed of thin, low contrast tube-like shapes. An interactive navigation has been proposed which leads to the definition of candidate venous paths toward the left ventricle. The joint measurements that can be carried out during the navigation (distance from reference entry points, diameters, angles) provide the means for a prior evaluation of path feasibility.

The 3D data sets examined so far were almost free from artefacts and blurring effects related to movement of the heart. However, these problems can become more critical when small structures like veins are concerned. The next generation of imaging device with an increase number of detectors and better motion-corrected reconstruction algorithms will minimize such problem in the near future. The next step is clearly the image guided intra-operative assistance of CRT. The poor contrast of fluoroscopic venous images makes this task very challenging for motion tracking and 2-D/3-D matching.

\section{Acknowledgements}

We would like to thank D Boulmier, C Leclercq, H Le Breton and all the physicians who have read and anatomically labelled the data sets. We are also indebted to B Le Bruno from Siemens France for her continuous assistance.

\section{References}

Abraham W.T, Fischer W.G, Smith A.L et al, Cardiac resynchronization in chronic heart failure, N.Engl.J.Med, 346, 1845-1853, 2002.

Alonzo C, Leclercq C, d'Allones F.R et al, Six years experience of transvenous left ventricular lead implantation for permanent biventricular pacing in patients with advanced heart failure: technical aspects, Heart, 86, 405-410, 2001.

Bardou.A, Auger P, Birkui P, Chasse J.L, Modeling of cardiac electrophysiological mechanisms: from action potential genesis to its propagation in myocardium, Crit.Rev.Biomed.Eng, 24, 141-221, 1996.

Bers.D, Cardiac excitation-contraction coupling, Nature, 415, 198-205, 2002.

Cazeau S, Leclercq C, Lavergne $\mathrm{T}$ et al, Effects of multisite biventricular pacing in patients with heart failure and intraventricular delay, N.Engl.J.Med, 344, 873-880, 2001. 
Coatrieux J.L, Toward the living human: the challenge of multimodal and multiscale processing and modeling, Multimodal Bio-Medical Systems Workshop, IEEE/NLM/NSF, Bethesda, Oct 2004.

Coatrieux J.L, Roux C, Biomedical Imaging IV, IEEE EMBS Book Series, IEEE Press, NJ, 2002.

Daubert J.C, Pitter P, Le Breton H et al, Permanent left ventricular pacing with transvenous leads inserted into the coronary veins, Pace, 21, 239-245, 1998.

De Bakker J.M.T, Hauer R.N.W, Simmens T.A, Activation mapping: unipolar versus bipolar recording, In: Zipes D.P, Jalife J Eds, Cardiac Electrophysioloy: from cell to bedsite, $3^{\text {rd }}$ Edition Philadelphia, Saunders, 1068-1078, 2000.

De Boer I H, Sachse F B, Mang S and Dössel O, Methods for Determination of Electrode Positions in Tomographic Images, International Journal of Bioelectromagnetism, 2, 2, 2000.

Garreau M, Simon A, Boulmier D, Guillaume H, Cardiac Motion Extraction in Multislice Computed Tomography by using a 3D Hierarchical Surface Matching Process, IEEE computers in Cardiology, Chicago, 2004.

Gepstein L, Hayan G, Ben-Haim S.A, A novel method for nonfluoroscopic catheter-based electroanatomical mapping of the heart: in vitro and in vivo accuracy results, Circulation, 95, 1611-1622, 1997.

Haigron P, Le Berre G, Coatrieux J.L, 3D Navigation in Medicine, Eng.Med.Biol.Mag, 15, 2, 70-78, 1996.

Haigron P, Bellemare ME, Acosta O, Goksu C, Kulik C, Rioual K, Lucas A. Depth-Map-Based Scene Analysis for Active Navigation in Virtual Angioscopy. IEEE Transactions on Medical Imaging , 23, 11, 1380-1390, 2004.

Hunter P.J, Nash M.P, Sands G.B, Computational electromechanics of the heart, in Computational Biology of the Heart, Wiley \& Sons, 345-407, 1996.

Kass D.A, Predicting cardiac resynchronization response by GRS duration, J.Am.Coll.Cardiol, 42, 2125-2127, 2003

Leclercq C, Kass D.A, Re-timing the failing heart: principles and current clinical status of cardiac resynchronization, J.Am.Coll.Cardiol, 39, 194-201, 2002.

Noble D, Winslow R.L, Reconstruction of the heart: network models of SA node-atrial interaction, in Computational Biology of the Heart, Wiley \& Sons, 49-64, 1997.

Quan W, Evans S.J, Hastings H.M, Efficient integration of a realistic two-dimensional cardiac tissue model by domain decomposition, IEEE Trans.Biomed.Eng, 45, 3, 372-385, 1998.

Roux C, Coatrieux J.L, Contemporary perspectives on Three Dimensional Biomedical Imaging, IOS Press, Amsterdam, 1997.

Rudy Y, Insights from theoretical simulations in a fixed pathway, J.Cardiovasc.Electrophysiology, 6, 294-312, 1995.

Schilling R.J, Peters .S, Davies D.W, Simultaneous endocardial mapping in the left ventricle using a noncontact catheter: comparison of contact and reconstructed electrograms during sinus rhythm, Circulation, 98, 887-898, 1998.

Schleich JM, Dillenseger JL, Andru S, Coatrieux JL, Almange C, Understanding normal cardiac development using animated models, IEEE Computer Graphics and Applications, 22, 14-19, 2002.

Sermesant M, Rhode K, Anjorin A et al, Simulation of the Electromechanical Activity of the Heart Using XMR Interventional Imaging, Proceedings MICCAI 2004, LNCS 3217, Springer-Verlag, 786-794, 2004

Taylor R, Lavallée S, Burdea G, Mosges R, Computer-integrated surgery: technology and clinical applications, MIT Press, 1996.

Virag N, Blanc O, Kappenberger L, Computer simulation and experimental assessment of cardiac electrophysiology, Futura Publishing, 2001. 